DIGITAL COMMONS
@ UNIVERSITY OF SOUTH FLORIDA

Journal of African Conflicts and

Peace Studies

Volume 2

Issue 2 : Memory, \& Peace Accords

Article 2

December 2015

\title{
La gouvernance des mémoires au Rwanda au travers du dispositif « ingando »: Une analyse critique des représentations sociales
}

Eric Ndushabandi

nseric26@yahoo.fr

Follow this and additional works at: https://digitalcommons.usf.edu/jacaps

Part of the African Studies Commons, Leadership Studies Commons, Other Political Science Commons, and the Other Social and Behavioral Sciences Commons

\section{Recommended Citation}

Ndushabandi, Eric (2015) "La gouvernance des mémoires au Rwanda au travers du dispositif « ingando »: Une analyse critique des représentations sociales," Journal of African Conflicts and Peace Studies: Vol. 2: Iss. 2, 37-61.

DOI: http://dx.doi.org/10.5038/2325-484X.2.2.2

Available at: https://digitalcommons.usf.edu/jacaps/vol2/iss2/2

This Article is brought to you for free and open access by the Open Access Journals at Digital Commons @ University of South Florida. It has been accepted for inclusion in Journal of African Conflicts and Peace Studies by an authorized editor of Digital Commons @ University of South Florida. For more information, please contact digitalcommons@usf.edu. 


\title{
La gouvernance des mémoires au Rwanda au travers du dispositif « ingando »: Une analyse critique des représentations sociales
}

\author{
Par Dr. Eric Ns. Ndushabandi \\ Docteur en sciences politiques \\ Université du Rwanda \\ nseri26@yahoo.fr
}

\begin{abstract}
Résumé
The objective of this paper is to understand the place of memory in the post conflict society reconstruction. The main objective of this paper is to understand how Rwanda is managing the past and the genocide memory through "Ingando", this kind of solidarity camps organized for all social categories. This paper builds its argument from findings of a doctoral research conducted on "Ingando". The Ingando framework constitutes one of major mechanisms through which the post genocide Rwandan government has attempted to create one "common interpretation of the past" or a "national collective memory" as part of the nation building agenda.
\end{abstract}

After human atrocities and violent conflicts, social representations of the past happen to converge with the official memory; but on another hand there are also noticeable divergences between the two and significant resistance from different groups. It is not always the official memory converges with "national collective memory" and the "official memory" is often conflicting with individual memories". How is all this happening in Rwanda? How do beneficiaries, most of who are university students, perceive this program and what impact is it having on them? Are there conceptual and practical challenges to this agenda as a public policy? This paper will attempt to bring responses to these questions and many more. This paper is articulated on three main points; the first one presents the subject and come back on the historical background. In the second point we define our approach and the main concept of memory and social representations. The last point presents main research findings and the critical conclusion.

Cette réflexion porte sur la reconstruction de la société et particulièrement sur la politique de la mémoire du génocide au Rwanda après 1994. Il s'agit principalement d'analyser la place de la mémoire dans la reconstruction post-conflit d'une société au travers d'un dispositif « ingando ». Celui-ci est compris comme un dispositif, vecteur de diffusion d'unité nationale et de la mémoire officielle pour une mémoire collective nationale. Nous articulons notre argumentation sur trois points. Dans un premier moment nous rappelons l'objet et les éléments de contexte, ensuite nous précisons notre approche et le sens des concepts ; et enfin nous nous présentons les principaux résultats de nos recherches.

\section{$1^{\circ}$ Objet et éléments de Contexte}

L'histoire du Rwanda ancien et colonial est soumise à des variations importantes dans la présentation des événements et des rapports sociaux, lesquelles apparaissent comme 
concurrentes et contradictoires. Pourtant, ces diverses lectures nourrissent encore les attitudes et les comportements tant dans le monde scientifique que dans le monde politique et social.

A l'aube de son indépendance en 1959, le Rwanda fut confronté au clivage Hutu-Tutsi qui s'amplifia au cours des années ultérieures. Au cours de cette année marquant la fin de la monarchie royale dite «tutsi », le Rwanda bascula dans une violence orchestrée par une élite «hutu » qui était parvenue à élaborer une idéologie au nom de «la révolution sociale ${ }^{1}$, laquelle était en faveur de la «majorité » hutu. Pour légitimer son plan d'extermination de ce que l'on nomme les «Tutsi ${ }^{2}$, cette élite « hutu » estimait que les Hutus avaient subi des humiliations dans le passé ${ }^{3}$. Elle se considérait comme ayant été longtemps exclue du pouvoir et ayant connu des vexations diverses ${ }^{4}$. Des actes d'incendies, de pillages et des massacres furent alors commis contre les Tutsis à l'échelle nationale. Ils débouchèrent sur une vague de réfugiés tutsis dans les pays limitrophes. Chaque tentative de retour au pays (1963-19701990) fut suivie de massacres parmi les Tutsis de l'intérieur, véritables boucs émissaires étant donné qu'ils partageaient «l'identité ethnique ${ }^{5}$ de ceux qui étaient désormais traité d'ennemis de la nation et d'étrangers. Cette stratégie de la part des Hutus extrémistes, qu'on qualifie de «Hutu power », consistait à rendre tous les Tutsis responsables de l'attaque du FPR (Front patriotique rwandais ?) ${ }^{6}$ contre le Rwanda. Elle servit de base pour justifier l'arrestation massive et le massacre de tous ceux qu'on considérait à tort ou à raison comme « complice » ou « ibyitso » en Kinyarwanda. La même stratégie a été utilisée durant la guerre qui opposa le gouvernement rwandais et le Front patriotique rwandais /Inkotanyi entre 19901994. Malgré la compagne militaire ${ }^{7}$, politique et médiatique ${ }^{8}$ qui insista sur la probable

\footnotetext{
${ }^{1}$ Lire, à ce propos, HARROY J.-P., Rwanda: souvenirs d'un compagnon de la marche du Rwanda vers la démocratie et l'indépendance, Bruxelles, Hayez, Académie des sciences d'Outre-mer, 1984.

${ }^{2}$ Lire « Les dix commandements des Bahutus », Kangura du 6 décembre 1990.

${ }^{3}$ On peut lire dans le journal Kangura du 16 mai 1991, (6), ce qui suit : " Les Tutsi nous ont trouvés au Rwanda, ils nous ont opprimés et nous l'avons supporté. Mais maintenant que nous nous étions sortis du servage et qu'ils veulent réinstaurer la chicote matinale, je pense qu'aucun Hutu ne pourra le supporter. La guerre que mène Gahutu est juste, c'est un combat pour la république. Que tous les Hutu le sachent, lorsque les féodaux arriveront au Rwanda, ils ne feront pas de distinction entre Hutu du Nord et ceux du Sud, qu'ils sachent que ce sera la fin à eux tous ".

${ }^{4}$ REYNTJENS F., L'Afrique des grands lacs en crise. Rwanda, Burundi : 1988-1994, Karthala, Paris, 1994. II faut dire que cette propagande a pris l'allure d'une guerre médiatique, comme on peut le lire dans le célèbre journal Kangura : "Les Tutsi nous ont trouvés au Rwanda, ils nous ont opprimés et nous l'avons supporté. Mais maintenant que nous étions sortis du servage et qu'ils veulent réinstaurer la chicotte matinale, je pense qu'aucun hutu ne pourra le supporter. La guerre que mène Gahutu est juste, c'est un combat pour la république. Que tous les hutu le sachent, lorsque les féodaux arriveront au Rwanda, ils ne feront pas de distinction entre hutu du Nord et ceux du Sud, qu'ils sachent que ce sera la fin à eux tous " voir, KANGURA N¹6, mai 1991, p.6 tel que cité par CHRETIEN, J.P. (s/dir), Rwanda. Les Médias du génocide, Karthala, Paris, 1995 p. 143. CHRETIEN J.P., op. cit.., p. 56.

${ }^{5}$ Ce concept sera défini plus loin. Voir MAALOUF A., Les identités meurtrières, Paris, Grasset, 1998, p.17.

${ }^{6}$ Le Front patriotique rwandais est un parti politique créé à partir de l'Ouganda en 1987-1988. Créé principalement par des exilés Tutsi chassés du Rwanda lors des évènements meurtriers de 1959, il fut connu sous le nom de «Rwandese Alliance for National Unity » (RANU). Les membres du FPR s'étaient fixés l'objectif principal de revendiquer le droit au retour dans leur pays d'origine. Après plusieurs tentatives d'inclusion et de retour négocié au Rwanda, les exilés Tutsi qui ont bénéficié du soutien des Tutsi et de quelques Hutu de l'opposition de l'intérieur ont enfin décidé de déclencher une guerre en octobre 1990 qui portat le FPR au pouvoir depuis juillet 1994 après une victoire militaire. Il a chassé du pouvoir le régime hutu présidé par Juvénal Habyarimana et ensuite le gouvernement intérimaire qui a organisé le génocide entre avril et juillet 1994. C'est donc dans un contexte de post-génocide que le FPR a entrepris le dur labeur de reconstruire le Rwanda après plusieurs décennies de conflits sanglants.

${ }^{7}$ La volonté affichée de se débarrasser des « complices » du F.P.R (Ibyitso) transparaît notamment à travers deux articles du journal gouvernemental “Imvaho”, rédigés par la journaliste Hélène Nyirabikari en janvier 1991
} 
reconquête du FPR, le gouvernement Habyarimana finit par céder à la pression de la communauté internationale qui exigeait un cessez-le-feu au FPR et l'ouverture de négociations. Malgré les efforts de résolution pacifique du conflit, le pays fut confronté à l'impasse qui se solda par le génocide d'avril à juillet $1994^{9}$.

Le dualisme identitaire hutu-tutsi qui avait conquis l'imaginaire social et politique des Rwandais primait sur toutes les autres formes d'identités collectives ${ }^{10}:$ il a rendu possible la mise en œuvre du génocide ${ }^{11}$ qui fut exécuté au nom de l'identité hutu contre l'identité tutsi.

Au lendemain du génocide, le Rwanda se présentait comme une société à la fois défigurée et aux multiples visages, une société de paroles et de silences. Une forme aigüe de désintégration sociale avait conduit à la mise en place de nouvelles catégories sociales issues du génocide : d'une part, les rescapés du génocide et, d'autre part, des familles décomposées par l'emprisonnement massif de leurs membres ainsi que des familles en recomposition après l'exil de 1959, 1963, 1990 et 1994.

Le 19 juillet 1994, le nouveau gouvernement rwandais mis en place à la suite d'une victoire militaire fut confronté au défi de la reconstruction nationale après un génocide sans précédent comptant environ un million de victimes parmi les Tutsis ainsi que parmi les Hutus opposés à

et en mars1992. NYIRABIKARI, H. « Mu Ruhengeri ni amahoro. Ariko umwanzi ntari kure. Mu Ruhengeri, ibyitso byagize uruhare... ! » Imvaho, no 880, 4-110 Gicurasi 1991.

${ }^{8}$ De nombreux médias d'État et journaux privés, dont Kangura qui a publié « Les Dix Commandements d'un Hutu ", (voir annexe $n^{\circ} 15$ ) ont joué un grand rôle dans la mobilisation des populations contre "l'ennemi » du pays. Entre 1990 et 1994, plusieurs partis politiques, dont le CDR et le MRND, ont sensibilisé la population sur le bien-fondé de la liquidation éventuelle de citoyens gênants.

${ }^{9}$ A propos de cette campagne médiatique qui a conduit au génocide, on peut lire chez Jean Pierre Chrétien ce qui suit : "Les prédictions rétrospectives sont trop faciles, mais l'innommable ne s'est pas inscrit dans un nondit. Littéralement, on pouvait le lire et l'entendre. Ce fut un génocide annoncé et revendiqué, et les textes que nous avons présentés en sont la chronique anticipée ou l'accompagnement médiatique. Les délations, les barrières, les machettes, les tortures et les meurtres, la mort, la haine et la cruauté sont présents dans les mots, dans les phrases, autant que suggérés entre les lignes de cette propagande " (CHRETIEN, J.P. (s/dir), Rwanda. Les Médias du génocide, Karthala, 1995, p. 379).

${ }^{10}$ En ce qui concerne les références claniques, voir notamment D'HELTEFELT, M., Les clans du Rwanda ancien. Eléments d'ethnosociologie et d'ethnohistoire, Bruxelles, Tervuren, 1971. Au sujet de l'identité nationale, voir SEBASONI S., Reconstruire une nation, Ed. Rwandaise, Kigali, 2007 et SHYAKA A., "Refondation de la rwandité : stratégie de résolution des conflits, de démocratisation et de développement durable du Rwanda», dans RUTEMBESA F. et alii, Identité et Citoyenneté, Butare, UNR, 2003. pp. 206-219.

${ }^{11}$ Nous adoptons, dès le départ, ce concept de génocide comme une catégorie juridique telle qu'elle a été définie par la résolution 955 du 8 Novembre 1994 du Conseil de sécurité de l'ONU qui reconnait le génocide commis au Rwanda en 1994. La résolution précise que le tribunal pénal international pour le Rwanda (TPIR) a été mis en place afin de juger les personnes responsables d'actes de génocide et d'autres violations graves du droit international humanitaire commis sur le territoire du Rwanda, ou par des citoyens rwandais sur le territoire d'Etats voisins, entre le $1^{\text {er }}$ janvier et le 31 décembre 1994 . A lire en ligne sur: www.un.org./dpts/dhl/dhlf/landmark_sc/topical.htm, consulté le 2 mai 2013. Dans son article 2, la résolution des Nations-Unies précise que le terme " génocide s'applique à des actes ci-après commis dans l'intention de détruire en tout ou en partie, un groupe national, ethnique, racial, ou religieux comme tel : a) Meurtre de membres du groupe, b) Atteinte grave à l'intégrité physique ou mentale de membres du groupe ; c) Soumission intentionnelle du groupe à des conditions d'existence devant entraîner sa destruction physique totale ou partielle; d) Mesures visant à entraver les naissances au sein d'un groupe. e) Transfert forcé d'enfants du groupe à un autre groupe ". Voir Convention de nations unies pour la prévention et la répression du crime de génocide adopté par l'assemblée générale le 9 décembre 1948.A lire sur: www2.ohchr.org/french/law/genocide.htm, consulté le 2 mai 2013. 
l'idéologie génocidaire ${ }^{12}$. Comment, dès lors, envisager la reconstruction d'une société fondamentalement déstructurée sur le plan social, politique et économique au lendemain du génocide ${ }^{13}$ ?

Face à cette réalité complexe, le gouvernement dit «d'union nationale » décida de faire renaître de ses cendres la société détruite par l'ethnisme et le génocide en mettant en place des mécanismes de dialogue dit national ${ }^{14}$ et de reconstruction. En particulier, il institua une politique de la mémoire comme stratégie de reconstruction de la société. Les initiatives du gouvernement rwandais dans le processus de gestion politique de la mémoire du génocide visaient à rétablir une société unie et réconciliée à partir d'institutions sociopolitiques favorisant la construction d'une «mémoire nationale ».

Au lendemain de la prise du pouvoir par le Front Patriotique Rwandais (FPR) et la fin du génocide, un vaste dispositif a été instauré avec l'aide des militaires et des organisations humanitaires ${ }^{15}$, afin d'évacuer les corps et autres restes humains jonchant les rues, les maisons, les églises, ainsi que les établissements publics et privés.

Les corps et autres restes des victimes sont inhumés en vitesse dans des fosses communes. Les mots pour expliquer ce qui s'est passé ne viennent pas encore aisément. C'est le dénuement total. Les confessions religieuses ouvrent timidement les portes des églises, suffoquant sous l'effet des odeurs des corps humains et des murs peints avec le sang des victimes. L'état dans lequel se trouve le pays est indescriptible; c'est pourquoi il existe très peu de discours avant les premières commémorations d'avril 1995. Pendant ces commémorations, le FPR montre qu'il a hérité non seulement d'un passé meurtrier, mais aussi, il évoque constamment les trente ans d'exil des Tutsi vers les pays limitrophes, (surtout en Ouganda, au Zaïre (RDC) et au Burundi), qui ont justifié leur campagne militaire et politique pour libérer le pays. Les Rwandais sont invités à ne pas oublier. La mémoire est décrite non seulement comme un devoir pour les victimes, mais aussi comme une pratique intrinsèquement liée à la nature humaine, et davantage encore comme une pratique traditionnelle rwandaise. Cette mémoire est en continuité avec la politique globale d'unité et de réconciliation nationale, laquelle commission était déjà prévue dans les accords de Paix

\footnotetext{
${ }^{12}$ Le consensus sur le nombre exact des victimes est loin de faire l'unanimité. L'ONU parle de 800000 victimes alors que les recensements censurés par le régime de Kigali évoquent plus d'un million de victimes. Voir Laure de Vulpian, Rwanda. Un génocide oublié ? Un procès pour mémoire, Ed. Complexe, Bruxelles, 2004, p.22.

${ }^{13}$ Pour plus de détails sur les différentes figures du Rwanda au lendemain du génocide, on peut lire le volume collectif de MOUTTAPA J., (s/dir), Rwanda. Pour un dialogue des mémoires, Albin Michel, Paris, 2007. II soulève la question des mémoires plurielles et, à notre connaissance, il constitue le seul ouvrage qui parle jusqu'à présent de dialogue des mémoires au Rwanda.

${ }^{14}$ Le gouvernement rwandais organise chaque année un sommet national qui réunit les acteurs tant politiques que civils depuis le niveau central jusqu'aux unités administratives décentralisées. Cette pratique remonte aux années 1995, lors des premières concertations organisées au village Urugwiro, la présidence de la République pour discuter des politiques publiques à adopter au lendemain de la victoire du FPR en 1994.

15 Ces commémorations consistent en des cérémonies d'inhumation des restes des victimes. Elles sont organisées par des églises et des groupes religieux, des rescapés et des autorités administratives au niveau des provinces et des " communes ». L'étude de Claudine Vidal analyse les " commémorations publiques " de 1995 à 2000 et montre que l'Etat a joué son rôle en ordonnant les commémorations officielles. Aucune structure officielle n'était chargée d'organiser des commémorations jusqu'à ce que l'Etat mette en place des commissions ad hoc chargées de ces cérémonies. Voir VIDAL C., " La commémoration du génocide au Rwanda : Violence symbolique, mémorisation forcée et histoire officielle ", Cahiers d'Etudes Africaines, n¹75, 2004, p.575. (1), (pp.575-592). En ligne sur : http://www. etudesafricaines.revues.org/4737, consulté le 13 décembre 2011.
} 
d'Arusha, signés le 4 août $1993^{16}$. Il s'agit de reconstruire une nouvelle manière d'interpréter l'histoire qui diffère de celle qui a été longtemps diffusée pendant les deux premières Républiques. Cette dernière est considérée comme une "fausse histoire " parce qu'elle fait des Tutsis et des Hutus des ennemis irréconciliables. Depuis 1994, et notamment avec la première commémoration du génocide en avril 1995, le gouvernement dit d'union nationale a affiché clairement sa volonté de bannir tout risque de répétition du génocide en favorisant la mémoire et non l'oubli $^{17}$. Ce discours assez volontariste se focalise sur le soutien et la compassion envers le rescapé du génocide chez qui il faut rallumer l'espoir de vivre. La mémoire se matérialise par la consolidation de certains instruments de la mémoire, comme l'exhumation des restes des victimes du génocide et leur inhumation accompagnée d'une célébration religieuse, la construction et l'entretien de sites mémoriaux, la consécration d'une semaine de deuil national l'organisation de débats publics et l'incitation à la recherche sur l'histoire du génocide. Une politique de la mémoire en tant que telle n'est formalisée que 13 ans après le génocide de 1994 par l'adoption d'une politique sectorielle de la mémoire du génocide $^{18}$. Elle est actuellement prise en charge par la Commission Nationale du Lutte contre le Génocide $(\mathrm{CNLG})^{19}$.

En 1999, le gouvernement a créé la Commission Nationale pour l'Unité et la Réconciliation $(\mathrm{CNUR})^{20}$ dans le but de se confronter au passé national. Cette commission avait pour fonction principale de promouvoir l'unité et la réconciliation des " Rwandais » par des débats publics, des formations de civisme, de patriotisme. Une lecture particulière de l'histoire nationale est réalisée au travers des «ingando ${ }^{21}$ organisés par la CNUR.

\footnotetext{
${ }^{16}$ Le préambule et particulièrement l'article 24 du protocole de paix du 30 octobre 1992 et des accords de paix signés le 4 août 1993, prévoient une commission d'unité et de réconciliation nationale qui a pour mission principale d'organiser les débats nationaux sur l'unité et la réconciliation, de préparer et de diffuser une information éducative destinée à la population et visant l'unité et la réconciliation nationales.

${ }^{17}$ Outre la prise en compte de la mémoire dans la reconstruction de la société rwandaise au lendemain du génocide, d'autres mécanismes transitionnels de justice ont été successivement adoptés. Il s'agit, d'une part, de la demande du Rwanda à la communauté internationale de mettre sur pied un Tribunal pénal international pour le Rwanda en 1995 et, d'autre part, l'adoption en 1998 du Fond d'assistance aux rescapés du génocide et l'activation de l'appareil judiciaire en proposant un mécanisme novateur de juridictions populaires Gacaca. Nous y reviendrons ci-dessous.

${ }^{18}$ MIJESPOC, Politique sectorielle de la mémoire du génocide des Tutsi, Kigali, 2008. Ce document fut rédigé par une équipe des chercheurs de l'Université Nationale du Rwanda dont nous faisions partie. Elle avait été commanditée par le Ministère de la Jeunesse, du Sport et de la culture (MIJESPOC), devenu Ministère du sport et de la culture (MISPOC) qui en assurait la tutelle. Cette Politique fut adoptée lors du Conseil des ministres du 12 mars 2008. Le titre du document non encore publié, mais qui régit les activités de la mémoire, est libellé comme suit : "Politiki yo kwibuka jenoside y’Abatutsi » (Politique de la mémoire du génocide des Tutsis). La notion de politique sectorielle est d'actualité dans la littérature en rapport avec l'analyse des politiques publiques. Une politique publique (sectorielle) est une tentative de gérer un rapport entre le secteur concerné et la société globale : c'est le rapport global-sectoriel (RGS) ; il s'agit donc en premier lieu de définir la place, le rôle et la fonction du secteur concerné par rapport à la société globale ou par rapport à d'autres secteurs. Voir MULLER P., Les politiques publiques, Presses Universitaires de France, Paris, $4{ }^{\text {ème }}$ édition : 2000, p.4-5; BOURICHE R., "Analyse des politiques publiques", Revue sciences Humaines, Algérie, Université Mentouri Constantine (Algérie), 2006, p.6; JOBERT B., MULLER. P., «L'Etat en action. Politiques publiques et corporatismes ", Revue française de science politique, 1988, Volume 38, Numéro 3, p. 433.

${ }^{19}$ Celle-ci est prévue par la Cconstitution de 2003 et régie par la loi numéro 09/2007 du 16 février 2007.

${ }^{20}$ Cette commission d'unité et de réconciliation nationale est prévue dans les Accords de Paix d'Arusha depuis 1993 et est régie par la loi 03/99 du 12/03/1999 créant la CNUR : elle fait partie intégrante de la nouvelle Cconstitution du Rwanda de 2003 en son article 178. La commission a son siège à Kigali et des représentations dans les provinces ; elle fait un rapport annuel au Président et au Sénat.

${ }^{21}$ Le concept "Ingando " dérive du verbe kinyarwanda " kugandika » qui signifie camper en plein air pour un groupe important de personnes ou encore camper pour reprendre le souffle après avoir parcouru de longues
} 
La CNUR n'ayant pas expressément dans ses attributions la tâche de préserver la mémoire et d'organiser des activités de commémoration, l'Etat s'est réservé, via le Ministère de la Culture, le monopole d'organiser les activités de commémoration du génocide jusqu'en $2008^{22}$. Cependant, le projet de politique sectorielle de la mémoire du génocide commis contre les Tutsis a été initié en 2006 ; il a été finalisé et adopté en 2008 sous la coordination du ministère ayant la culture dans ses attributions, le MIJESPOC. Cette politique de la mémoire a été proposée pour validation à un certain nombre d'acteurs politiques et à des organisations de la société civile. Ces acteurs regroupaient à la fois les institutions publiques et privées comme le Ministère de l'Administration locale, la CNUR, la Commission Nationale des Droits de la Personne, l'Institut des Musées Nationaux du Rwanda, l'Organe National des Juridictions Gacaca, le Fonds d'Assistance des Rescapés du Génocide, l'association mémoire ou IBUKA, et l'association des veuves du génocide (AVEGA Agahozo).

Avant d'être adopté par la décision du Conseil des ministres du 12 mars 2008, le Projet de politique de la mémoire du Génocide a été analysé et approuvé par un comité interministériel de coordination (C.I.C) composé des secrétaires généraux et des ministres, successivement le 13 juin 2007 et le 03 octobre $2007^{23}$. Depuis 2008, la mise en œuvre de cette politique est confiée à la Commission Nationale de Lutte contre le Génocide (CNLG), laquelle a pourmission principale de prévenir et de lutter contre le génocide et son idéologie ainsi que de gérer ses conséquences. Depuis 2010, une nouvelle approche a été adoptée : elle prévoit une décentralisation des activités de la commémoration au niveau local, c'est-à-dire au niveau de l'unité administrative la plus basse du Rwanda appelée " umudugudu » qu'on peut traduire par «village »? L'approche de politique de la mémoire au niveau de la base, laquelle rappelle la «Grassroots memorials ${ }^{24}$, renvoie au phénomène courant qui considère la mémoire comme une forme d'action sociale dans l'espace public. Généralement, cette remémoration se fait sur les sites mêmes où les événements traumatiques ont eu lieu. Ce processus de rémémorisation au niveau local désigne un processus par lequel les groupes sociaux transforment leurs perceptions en une action collective en créant une mémoire temporaire afin d'améliorer une situation particulière ${ }^{25}$. Ceci manifeste une volonté d'instaurer un processus

\footnotetext{
distances avec ses armes sur la tête. Dans ce sens, le concept ingando renvoie au contexte de guerre. Dans le contexte actuel, le concept "ingando", se traduit parfois par "camp de solidarité » ou "camp de rééducation " selon son référent: étudiants ou ex-forces armées rwandaises qui reviennent de l'exil. Les ingando constituent une activité importante pour la CNUR. Voir le rapport de la CNUR, Impact of the solidarity camps/INGANDOs on Unity and Reconciliation of Rwandans". 1996 - 2006, Kigali, 2006, p.17-18. Les ingando ont été conçus initialement pour favoriser la réintégration des réfugiés. Dans ce cas, les ingando sont considérés comme des camps de rééducation ou de réintégration. Ils ont été étendus à différentes catégories de la population telle que les fonctionnaires de l'Etat, les étudiants et les prisonniers libérés. Dans ce deuxième cas, les ingando sont davantage considérés comme des camps de solidarité. L'écart entre les deux n'est pas très important: dans les deux cas, il s'agit de camps de rééducation. Ils ont pour objectif de transmettre non seulement ce que l'Etat considère comme "la nouvelle et vraie version de l'histoire ", mais aussi un programme de diffusion des politiques publiques pour lesquelles on demande l'adhésion de la population. Nous y reviendrons plus loin. Voir CNUR, s/dir de SHYAKA A.), Le Conflit Rwandais, Origines, Développement et stratégies de sortie, Kigali, 2006, p.41. En ligne sur cec.rwanda.free.fr/document/doc/Doc-Rwanda/ Le conflitrwandais-Anastase-Shyaka.pdf, consulté le 4 mai 2013

${ }^{22}$ C'est-à-dire à la création de la CNLG qui s'y consacre exclusivement.

${ }^{23}$ Cabinet paper on National memory policy, Kigali, 2008.

${ }^{24}$ MARGRY P. et SANCHEZ CARRETERO C., "Rethinking memorialization. The concept of grassroots memorials, in MARGRY P. et SANCHEZ CARRETERO C., Grassroots memorials. The politics of memorizing traumatic deaph. Mapping Cultural history, Vol. 12, Barghahn Books, 2011, p.1-3.

${ }^{25}$ Ibidem, p.3.
} 
de construction d'une «mémoire collective ${ }^{26}$. Selon la note politique de la $\mathrm{CNLG}^{27}$, cette décentralisation des activités commémoratives consolide la participation des citoyens aux activités de commémoration ${ }^{28}$. L'appropriation au niveau local est préférée à une commémoration au niveau des provinces : celle-ci est moins propice à l'appropriation de la mémoire par les citoyens vu la distance par rapport aux lieux de massacres.

Dans son rôle de mobilisateur, de formateur et d'informateur, la CNUR organise les «ingando» via son département chargé de l'éducation civique. Celui-ci a pour tâches principales la préparation des syllabus ou manuels de formation, la formation elle-même et la mobilisation des masses populaires en faveur de l'Unité nationale et de la « reconstruction » d'une « nation prospère ${ }^{29}$.

Cet papier est une réflexion sur le cas d'étude des « ingando » au Rwanda en tant que vecteur privilégié de diffusion de la mémoire officielle. Son originalité tient au fait que les «ingando » sont abordés en tant que vecteur concret de la politique de la mémoire au Rwanda.

Dans un contexte où les politiques mémorielles font l'objet d'analyses de plus en plus nombreuses ${ }^{30}$, nous évaluons ici les effets que produisent les ingando en tant que vecteur de diffusion de la politique de la mémoire du passé et de celle du génocide.

\footnotetext{
${ }^{26}$ Au sens de Maurice Halbwachs la "de mémoire collective ", dans le cadre sociaux de la mémoire, Albin Michel, Paris, 1994. Le concept de " mémoire collective » sera abordé largement plus loin dans la partie théorique. Prenant en compte toute la querelle entre sociologues, historiens et psychologues, la mémoire collective est ici comprise au sens de Maurice Halbwachs, comme l'ensemble des souvenirs partagés par un groupe. De ce fait la mémoire collective contribue à façonner la vie d'un groupe. Ce groupe peut être ethnique, religieux, ou national. La définition de la "mémoire collective" peut donc varier selon le cas auquel on l'applique. Pour une mémoire collective partagée par un groupe national suppose qu'on mette l'accent sur l'unité de la nation, réifiée, qui pense et se souvient. Ce qui suppose qu'on affirme la transcendance du groupe dont les représentations collectives s'imposent nécessairement aux individus. Lire M-C LAVABRE, « Du poids et du choix du passé. Lecture du syndrome de Vicy », dans PESCHANSKI D., POLLAK M., ROUSSO H., (s/dir), Histoire politique et sciences sociales, Edi complexes, Paris, 1991, 265-278.

${ }^{27}$ La CNLG envoie une note comprenant les orientations (amabwiriza), les thèmes annuels de commémoration. Cfr CNLG, Rapport yo Kwibuka ku ncuro ya 15 jenoside yakorewe abatutsi, 2009, p.18.

${ }^{28}$ Cette nouvelle orientation comporte de nombreux enjeux. Ils ont été présentés dans un article que nous avons publié récemment: NDUSHABANDI N. E., " Figure et identité de la victime à travers l'Association des Etudiants et Elèves Rescapés du Génocide, (AERG), dans "Génocide : Les figures de la victime ", paru dans la revue La pensée et les Hommes, $n^{\circ} 85,2012$, pp.135-145.

${ }^{29}$ Rwanda : CNUR, Structure fonctions et programmes de la CNUR, en ligne sur http://www.grandslacs.net/doc/2385.pdf, p.2-3, Mars, 2009.

${ }^{30}$ L'article est de Véronique Charléty articule les usages de l'histoire et de la mémoire collective. Son ouvrage constitue une recherche dense et originale sur les politiques mémorielles en vigueur sur le territoire allemand dans la seconde partie du $20^{2}$ ème siècle. L'enjeu de cet ouvrage est d'illustrer les différentes trajectoires mémorielles et les conflits d'interprétation à l'égard du passé, d'analyser les événements non pour eux-mêmes mais dans leur dimension construite, dans l'effacement et la résurgence de leur signification. L'ouvrage illustre les mémoires désaccordées sur le continent européen au travers des débats suscités par une exposition, un projet de loi ou un lieu de mémoire. Voir CHARLETY V., "Jeux de mémoire : un enjeu pour l'Europe " dans Revue Française de science politique, Vol 161, n², Avril 2011, p.305-308. MAUREL M.C., MAYER F., (S/dir), L'Europe et ses représentations du passé. Les tourments de la mémoire, L'Harmattan, Paris, 2008. Voir encore ZEHFUSS M, Wounds and Memory, The politics of war in Germany, Cambridge, Cambridge University Press, 2007, 294p.; CHAUMONT, J. M. La concurrence des victimes, génocide, identité, reconnaissance, La Découverte, Paris, 1997 ; LEFRANC S, « Les victimes écrivent leur histoire. Introduction », Raisons politiques, 30 Mai, 2008, p5-19.
} 
Le fil conducteur de cette réflexion s'articule autour de la question principale de savoir quelle est la place de la mémoire dans le processus de reconstruction de la société post conflit, au Rwanda, au travers du dispositif des ingando ? En quoi les ingando constituent-ils un vecteur de diffusion de la mémoire officielle et quelle est la nature et la portée de cette mémoire officielle ? Quels effets produisent les ingando sur les représentations sociales du passés des participants?

Qu'est-ce qui explique le rapprochement et/ ou la résistance des représentations sociales par rapport à la mémoire officielle diffusée au travers des ingando ? A quelle condition la mémoire officielle peut-elle déboucher sur une mémoire collective nationale, c'est-à-dire le rapprochement des mémoires collectives des individus et des groupes ? Enfin, dans quelle mesure, les ingando peuvent-ils constituer un dispositif adéquat pour faire émerger une mémoire véritablement collective moyennant?

Les ingando sont ici abordés comme un «dispositif » au sens de Bruno Latour qui considère que le concept de «dispositif» convient le mieux pour désigner tous ces assemblages sociotechniques d'humains et de non-humains auxquels s'intéressent ces sociologues pour décrire les " programmes d'action ${ }^{31}$, "d'apprécier l'évolution de l'appareil administratif d'État à travers l'étude du travail bureaucratique tel qu'il se fait, au moment où il se fait et du point de vue de ceux qui le font $»^{32}$.

Il s'agit d'analyser les effets que produit une politique publique. Les politiques mémorielles font de plus en plus objet d'analyse ${ }^{33}$. Pour analyser les effets directs ou indirects d'un dispositif sur les représentations sociales, on peut partir des objectifs tels que fixés sur papier ou des réalisations concrètes sur terrain ou alors de son impact sur les usagers.

Deux approches ont été privilégiées dans cette réflexion, l'approche cognitive et les approches pragmatiques.

30 JOHNSTON W, Postmodernisme et bimillénaire. Le recueil des universitaires de la culture européenne, PUF, Paris, 1992.

${ }^{31}$ Lire LATOUR, B., Petites leçons de sociologie des sciences, Paris, Seuil, 1996.

32 Lire WELLER J-M., L'Etat au Guichet: sociologie cognitive du travail et modernisation administrative des services publics, Desclée de Brouwer, Paris, 1999. Pour cette notion d'Etat lire aussi Lire WELLER J-M., L'Etat au Guichet: sociologie cognitive du travail et modernisation administrative des services publics, Desclée de Brouwer, Paris, 1999.

MOULIERE M., "A propos des interactions au guichet de l’Etat », dans Mouvements, n¹5-16.La Découvertes, Paris 2001/3, en ligne sur: http://www.cairn.info/revue-mouvements-2001-3-page-190.htm , 20 Décembre 2011.

${ }^{33}$ L'article est de Véronique Charléty fait une articulation entre les usages de l'histoire et la mémoire collective. Son ouvrage constitue une recherche dense et originale sur les politiques mémorielles en vigueur sur le territoire allemand dans la seconde partie du $20^{\text {ème }}$ siècle. L'enjeu de cet ouvrage est d'illustrer les différentes trajectoires mémorielles et les conflits d'interprétation à l'égard du passé, d'analyser les événements non pour eux-mêmes mais dans leur dimension construite, dans l'effacement et la résurgence de leur signification. L'ouvrage illustre les mémoires désaccordées sur le continent européen au travers des débats suscités par une exposition, un projet de loi ou un lieu de mémoire. Cfr CHARLETY V., " Jeux de mémoire : un enjeu pour I'Europe " dans Revue Française de science politique, Vol 161, n², Avril 2011, p.305-308. MAUREL M.C., MAYER F., (S/dir), L'Europe et ses représentations du passé. Les tourments de la mémoire, Paris L'Harmattan, 2008. On peut lire ZEHFUSS M, Wounds and Memory, The politics of war in Germany, Cambridge, Cambridge University Press, 2007, 294p.

CHAUMONT, J. M. La concurrence des victimes, génocide, identité, reconnaissance, La Découverte, Paris, 1997. LEFRANC S, « Les victimes écrivent leur histoire. Introduction », Raisons politiques, 30 Mai, 2008, p519.

${ }^{33}$ JOHNSTON W, Postmodernisme et bimillénaire. Le recueil des universitaires de la culture européenne, PUF, Paris, 1992. 
L'approche cognitive que nous avons adoptée dans cette analyse s'intéresse plus aux acteurs à plusieurs égards. Dans cette analyse de politique publique, ce sont les approches cognitives qui nous permettent de comprendre les valeurs et les logiques justificatives de choix d'action des acteurs politiques dans la mémoire du génocide au Rwanda. L'approche cognitive de l'analyse des politiques publiques est une approche par «vision du monde partagée ».

Les approches pragmatiques préconisent, enfin, que la décision doit être réinterprétée, elle n'est pas le moment clé, il est plus intéressant dans notre cas de montrer qu'une analyse des justifications de l'action publique témoigne des présupposés normatifs et de cadres d'interprétation de l'action. C'est pourquoi, il est impératif de s'intéresser à la politique telle que écrite sur papier mais aussi la politique en acte.

Le travail d'analyse des effets d'une politique publique comporte un aspect pratique. C'est pourquoi nous avons fait une analyse documentaire portant principalement sur le manuel de formation des ingando comme document de référence dans l'analyse du contenu des représentations officielles du passé. En plus de ce manuel, nous prenons en compte tous les matériaux documentaires des formateurs et de façon complémentaire quelques discours officiels qui traduisent cette vision. Pour mesurer les représentations sociales et les représentations officielles nous avons fait recours à plusieurs techniques de recherche dont l'observation directe dans les ingando, le questionnaire, l'entretien et le groupe de discussion dirigé. L'observation directe a été articulée sur quatre questions centrales : qui parle, A qui parle-t-on ? De quoi parle-t-on ? Et enfin Comment et «pour quoi »? Cette perspective nous permet de prendre au sérieux tous les acteurs.

\section{$2^{\circ}$. Quelques concepts de base : Mémoire individuelle, mémoire officielle et mémoire collective (nationale)}

La mémoire individuelle ou mémoire vive chez Marie-ClaireLAVABRE ${ }^{34}$ et Van YPERSELE $^{35}$ est faite des souvenirs des membres du groupe qui sont vécus et transmis « horizontalement », sans intervention des discours politiques qui façonnent quant à eux une sorte de mémoire officielle. Entre les mémoires vives Qu'est-ce qu'une mémoire officielle, quels sont ses déterminants et ses limites ?

De prime abord, la mémoire officielle apparaît comme l'expression d'un pouvoir politique qui s'exprime au cours des commémorations des événements qui ont le plus marqué l'histoire nationale. "La mémoire officielle est le recueil des faits qui sont supposés avoir le plus marqué la mémoire des hommes, des faits qui ont contribué à changer les nations ou les institutions. Lus dans les livres ou vécus, les événements passés sont choisis, rapprochés, suivant des nécessités ou des règles qui ne s'imposaient pas aux hommes qui en ont longtemps gardé le dépôt. ${ }^{36}$.

Cette mémoire est par nature sélective et est soumise aux préférences et aux impératifs des hommes politiques. La mémoire officielle, est une mémoire produite ou promulguée, par les

\footnotetext{
${ }^{34}$ LAVABRE, M.-C., Le fil rouge : sociologie de la mémoire communiste, Presses de la Fondation nationale des sciences politiques, Paris, 1994.

${ }^{35}$ Van YPERSELE, L., "Les mémoires collectives », dans van YPERSELE L., (s/dir.), Questions d'histoire contemporaine: conflits, mémoires et identités PUF, Paris, 2006, pp. 191-201.

${ }^{36}$ HALBWACHS M., Op. Cit., (1968), p. 60.
} 
dirigeants d'un Etat. Elle est destinée à devenir hégémonique, à être partagée par la collectivité tout entière. Elle s'actualise dans différentes institutions ou productions collectives, telles que les commémorations, les monuments, les manuels scolaires, les discours politiques, etc. Halbwachs nous propose la définition suivante : "la mémoire officielle est la suite des événements dont l'histoire officielle conserve le souvenir. Ce ne sont pas ces cadres qui constituent la mémoire collective, car entre l'individu et la nation, il y a bien d'autres groupes, plus petits qui ont leur mémoire et dont la transformation agit plus directement sur la vie et la pensée de leurs membres (...) » ${ }^{37}$.

La contribution de Valérie Rosoux est très précise quant au caractère aux déterminants et aux limites de la mémoire officielle. "Le caractère sélectif et fluctuant de la mémoire n'est pas un attribut négatif, mais fonctionnel - ou inhérent - de tout recours au passé. Il résulte du fait que la mémoire ne se réduit pas à une répétition ou à une conservation pure et simple du passé, mais qu'elle s'emploie constamment à réorganiser le passé. La mémoire officielle repose tout entière sur ce mécanisme d'ajustement du passé au présent. Bien que les éléments relatés soient souvent éloignés dans le temps, l'ensemble des évocations officielles du passé ne concerne en réalité que peu le passé : elles informent essentiellement sur le présent. C'est bien en fonction d'objectifs politiques présents que la mémoire officielle sélectionne les éléments historiques qu'elle juge pertinents. A cet égard, les tenants de la mémoire officielle voient davantage le passé comme un auxiliaire commode à leurs intentions, plutôt que comme un récit immuable $»^{38}$.

Ce remaniement de la mémoire est fait par souci réel ou affiché de la volonté de l'Etat de promouvoir le rapprochement entre les communautés en conflits. Dans ce cas, les autorités politiques peuvent tenter d'apaiser les souvenirs liés au passé national conflictuel et d'encourager le rapprochement, "d'accentuer tel ou tel autre aspect du passé national harmonieux» et enfin, «de méconnaitre ou de reconnaitre la mémoire des groupes adverses ${ }^{39}$.

Dans tous les cas, ni le souvenir, ni l'oubli ne peuvent s'imposer à l'ensemble de la communauté, car selon Valérie Rosoux, «le discours sur le passé n'influence que jusqu' à un certain point les représentations partagées par les individus. Les écarts qui peuvent exister entre l'interprétation officielle d'un événement et les perceptions qui prévalent au même moment dans l'ensemble de la société témoignent de la résistance de la mémoire vive par rapport à la mémoire officielle ${ }^{40}$.

La «mémoire officielle » c'est celle que les instances de l'Etat, tentent par la glorification, la mythification ou l'occultation, de forger et de maintenir et qui coïncide avec l'ambition de construire «une identité et une mémoire nationale». Cette mémoire officielle s'adresse pourtant aux «mémoires de groupes », celles partagées par des classes sociales, des groupes ethniques, des partis politiques, des associations, des militants d'une cause, des volontaires de la lutte contre tel ou tel oubli - ce qui suppose aussi, évidemment une mémoire sélective. Elles fonctionnent aussi dans une logique de construction identitaire. Ces mémoires des groupes se nourrissent des expériences individuelles, (mémoires individuelles) et de la «mémoire

\footnotetext{
${ }^{37}$ HALBWACHS M., op.cit, (1968), p.67.

${ }^{38}$ ROSOUX V., «Le Rôle de la mémoire en politique étrangère. Essai de théorisation », dans, TANGY. DE

WILDE et L. SPETSCHINSKY L., Les relations entre l'UE et la Fédération de Russie, UCL, 2000. En ligne sur http://www.uclouvain.be/cps/ucl/doc/euro/documents/Rosoux__ue_russie.pdf , 12 Décembre 2012, p.2.

${ }^{39}$ Ibidem.p4-5.

${ }^{40}$ Ibidem., p.5.
} 
savante », celle des historiens ${ }^{41}$. La mémoire officielle et la mémoire individuelle, peuvent se compléter, coexister en harmonie, mais il n'est pas rare qu'elles entrent en conflit ${ }^{42}$. La mémoire individuelle peut résister à la mémoire officielle par ce qu'elle ne colle pas à la réalité ou ne rejoint pas les attentes des individus. Or, comme le dit Valérie Rosoux, «cette résistance varie essentiellement en fonction de trois facteurs, non exclusifs l'un de l'autre : la plus ou moins grande fidélité de l'interprétation officielle de la réalité du passé (1), le niveau de correspondance avec les attentes de la population (2) et le degré de légitimité du commémorateur (3)... Comme toutes les formes de mémoire, la mémoire officielle n'est pas objective. Elle colore le passé à son avantage... La mémoire vive, aussi malléable soit-elle, résiste à la négation pure et simple des événements qui ont été vécus ou transmis par la population. Le décalage qui existe entre la mémoire officielle et la mémoire vive ne résulte pas seulement du degré de liberté qui est pris par rapport aux faits. Certaines représentations officielles du passé remportent l'adhésion de la population, alors même qu'elles ne respectent pas la réalité du passé... Les phénomènes de résistance rencontrés par l'interprétation officielle dépendent également d'un troisième facteur, en général étroitement lié au deuxième : la légitimité populaire du garant de la mémoire officielle. En effet, le passé plus ou moins hérö̈que du commémorateur peut lui aussi déterminer le degré d'adhésion manifestée par la population $»^{43}$.

La grille nous propose une manière d'interroger le degré de rapprochement entre ce qui est enseigné dans les ingando et la vérité historique tant revendiquée dans les représentations sociales.

Cette grille d'analyse nous permet ensuite de nous interroger sur le degré de correspondance ou de résistance entre les représentations sociales des participants et les représentations officielles transmises dans les ingando Enfin, la grille nous propose d'analyser le degré de légitimité et les compétences des orateurs qui interviennent dans les ingando et surtout la manière dont leurs expériences personnelles influent sur la manière dont les ingando sont faits et l'émergence d'une mémoire collective nationale.

Nous conclurons avec Marie-Claire Lavabre qui affirme que «la mémoire officielle et la mémoire vive s'articulent et se nourrissent l'une et l'autre. Elles se constituent simultanément, selon le schéma d'une instauration mutuelle et croisée. Nombre de souvenirs individuels sont encadrés par des récits collectifs, renforcés par des commémorations et relatés dans les cours d'histoire. A l'inverse, ces institutionnalisations du passé n'ont de sens que rapportées aux souvenirs et aux identifications individuelles $»^{44}$.

C'est pourquoi, il est important de de s'interroger sur leur recoupement, le rapprochement ou les décalages entre les deux ${ }^{45}$. Notre thèse trouve donc ici son principal fondement théorique.

\footnotetext{
${ }^{41}$ Lire FRANK R., Op.cit.

${ }^{42}$ LICATA L., KLEIN O., et GELY R., « Mémoire des conflits, conflits de mémoires : une approche psychosociale et philosophique du rôle de la mémoire collective dans les processus de réconciliation intergroupe », dans Social Science Information, Sage Publications, Vol 46/N4, Décembre, 2007 www.sagepub.com, consulté le 3 Août 2011.

${ }^{43}$ ROSUX V., Op.cit.p.5-7.

${ }^{44}$ LAVABRE M-C., Le fil rouge. Sociologie de la mémoire communiste, Presses de la fondation nationale des sciences politiques, Paris, 1994, p.18.

${ }^{45}$ Lire ROSOUX V., « La réconciliation franco-allemand : crédibilité et exemplarité d'un « couple à toute épreuve »? », dans Cahiers d'histoire. Revue d'histoire critique,(en ligne), 100, 2007, mis en ligne le 01 janvier 2010, consulté le11 mars 2011, p.2.
} 
Pour qu'il y ait recouvrement, la mémoire travail de mémoire et ici requis, sans quoi, la mémoire nationale semble hypothétique. D'où viendrait-elle ?

La mémoire officielle donc n'est pas une mémoire collective nationale, car entre les deux, il y a un écart significatif. Si les groupes restreints au sein d'une nation peuvent résister à la mémoire officielle pour des raisons évoquées plus haut, cette mémoire ne peut se hisser et s'ériger en une mémoire collective nationale, c'est-à-dire en tant que groupe national que moyennant ce que Paul Ricœur appelle « un travail de mémoire ${ }^{46}$. Cette approche de travail de mémoire, postule qu'une société ne peut exister qu'à condition d'avoir en commun un certain consensus sur le sens de l'histoire nationale. Pour que cette mémoire collective nationale émerge, il est incontournable que les membres de la communauté nationale se perçoivent comme une entité cohérente. La société mobilise un imaginaire collectif qui comprend notamment une certaine perception du passé et une certaine mémoire à entretenir vis-à-vis de certains faits et évènements. C'est ce que confirme Valérie Rosoux en montrant la spécificité de cette initiative entreprise par les Etats commémorateurs: "le travail de mémoire ne porte pas sur les faits eux-mêmes (que nul ne peut nier), mais sur le sens qui leur est attaché. C'est en jouant sur ce sens que les protagonistes essaient d'établir un récit qui puisse favoriser le rapprochement des différentes parties. Son but n'est pas d'établir la vérité avec un grand "V», mais de relire le passé à l'aune de la coopération recherchée, forme d'un compromis entre plusieurs représentations. Elle se démarque ainsi des logiques de revanche et d'oubli. La prise en considération de plusieurs points de vue ne signifie pas que toutes les perspectives soient pour autant équivalentes. Reconnaître la pluralité des représentations du passé ne remet pas en cause l'existence d'une réalité en deçà de ces représentations. L'élaboration d'une mémoire partagée ne se fonde pas sur le relativisme, mais sur l'idée qu'une histoire commune est possible $\gg^{47}$.

Or la mémoire officielle, dans sa nature sélective, peut choisir d'ignorer certains faits d'accentuer d'autres ou alors d'affronter les vérités relatives des groupes et des individus pour trouver ensemble une trame plus ou moins commune à tous les acteurs de la société. Cette trame commune ne signifie pas cependant que toutes les vérités se valent, au contraire les membres d'un groupe national peuvent se mettre d'accord sur le sens de certains faits et de partager un sens commun de l'avenir du groupe national.

En application à notre cas d'étude, les propos de Licata, Klein et Gély, sont très explicites pour le cas du Rwanda. "La mémoire vive concerne les souvenirs des membres du groupe qui sont vécus et transmis "horizontalement", sans intervention des discours dominants. La façon dont les récits du génocide tutsi se sont transmis (souvent oralement) au sein des familles et $d u$ voisinage relève de cette catégorie. Tandis que la mémoire officielle, en revanche, est une mémoire produite, ou promulguée, par les dirigeants du groupe. Elle est destinée à devenir hégémonique, à être partagée par la collectivité tout entière. Elle s'actualise dans différentes institutions ou productions collectives, telles que les commémorations, les monuments, les manuels scolaires, les discours politiques, etc. Ces deux catégories de mémoires peuvent se compléter, coexister en harmonie, mais il n'est pas rare $q u$ 'elles entrent en conflit $»^{48}$.

\footnotetext{
${ }^{46}$ RICOEUR, P. Temps et Récit, III, Paris, Seuil, 1985, p. 411

${ }^{47}$ ROUSOUX V., Op.cit., (2000), p.11-12.

48 LICATA L., KLEIN O. et GELY R., «Mémoire des conflits, conflits de mémoires : une approche psychosociale et philosophique du rôle de la mémoire collective dans les processus de réconciliation intergroupe », dans Social Science Information, Vol. 46/No 4, Décembre, 2007, en ligne sur http://www.ulb.ac.be/psycho/psysoc/Papers/licata,\%20Klein\%20\&\%20G\%E91y\%20-
} 
Dans le cas où il y a le conflit entre mémoire officielle et mémoire individuelle, le fossé se creuse et les conflits peuvent resurgir à tout moment. Le travail de mémoire consisterait alors à opérer le rapprochement entre les différentes mémoires dont la mémoire officielle d'une part et les mémoires individuelles et collectives d'autre part. C'est donc dans ce sens que nous faisons recours à ces concepts pour comprendre les dynamiques mémorielles au niveau individuel social ou collectif. Qu'est-ce que la mémoire collective?

Contrairement à la démarche adoptée dans la transmission d'une mémoire officielle transmise d'en haut à travers des discours officiels, des commémorations, des manuels scolaires et des manuels de formation, le travail de mémoire cherche à prendre en compte le conflit d'interprétations qui résulte immanquablement d'un événement tel que la guerre ou le génocide. L'objectif d'un travail de mémoire est de reconnaître la pluralité des interprétations du passé. A ces propos, Valérie précise que dans le travail de mémoire, «il ne s'agit plus de mettre en avant une vision martyrologique ou édulcorée du passée, mais de prendre en charge le passé dans sa complexité et se contradictions. Le travail de mémoire ne porte pas sur les faits eux-mêmes, (que nul ne peut nier) mais sur le sens qui leur est attaché. C'est en jouant sur le sens que les protagonistes essaient d'établir un récit qui puisse favoriser le rapprochement des différentes parties. Son but n'est pas d'établir la vérité avec un grand» $V »$ mais de relire le passé à l'aune de la coopération recherchée ».La mémoire dont il est ici question prend la forme d'un compromis entre plusieurs représentations. Elle se démarque ainsi des logiques ici est des logiques de revanche et d'oubli ${ }^{49}$.

Que signifie la prise en compte de toutes ces divergences ? Quels sont les critères nécessaires pour établir une vérité historique réconciliatrice? Le travail de mémoire procède par l'intégration des différentes interprétations et par la recherche d'un sens plus ou moins acceptable par tous. Ce qui ne signifie ni une simple juxtaposition «des vérités» relatives, ni la recherche de toute la vérité, et encore moins une imposition de la «Vérité », mais bien plus, une prise en compte des divergences dans la tolérance, en vue d'un projet national commun. Dans cette perspective le travail de mémoire ne consiste pas à ramener à surface tous les souvenirs douloureux ni de d'ignorer purement et simplement certains faits du passé, mais bien au contraire de le prendre en compte dans la construction d'une mémoire collective nationale.

\section{$3^{\circ}$. Principaux résultats}

Les ingando étant entendus comme un dispositif à travers lequel l'Etat transmet une certaine mémoire officielle dans l'objectif de faire émerger une mémoire dite «nationale » ou collective. Voici en bref les résultats les plus saillants de notre recherche :

- Le pragmatisme et la discontinuité dans la mise en ouvre des politiques publiques

A partir de l'analyse de l'action de l'Etat dans la gestion du passé, nous avons constaté qu'il y a une discontinuité très significative dans le processus de mise en œuvre des politiques publiques en rapport avec la gestion du passé au Rwanda. De la conception du dispositif

\%20M\%E9moire\%20des\%20conflits,\%20conflits\%20de\%20m\%E9moires.pdf , consulté le 15 décembre 2012. Voir aussi, LAVABRE M-C., Le fil rouge : sociologie de la mémoire communiste, Presses de la Fondation nationale des sciences politiques, Paris, 1994. VAN YPERSELE L., «Les mémoires collectives », dans VAN YPERSELE L., (Ed.), Questions d'histoire contemporaine: Conflits, mémoires et identités (pp. 191-201), Presses Universitaires de France, Paris, 2006.

${ }^{49}$ ROSOUX V, Le rôle de la mémoire en politique étrangère. Essai de théorisation, op.cit., p11-12. 
ingando, à la mise en place de la CNUR, du contenu du programme et de la politique qui la régit, il y a des écarts très significatifs ${ }^{50}$ qui ont un impact négatif sur la continuité de l'action publique. Ce qui justifie en partie, l'absence de mécanismes concrets, réguliers, homogènes et rigoureux pour évaluer les ingando au niveau de l'impact sur les représentations sociales.

\section{- Les ingando constituent « un cadre militarisé et redouté »}

Les résultats de nos recherches montrent bien que les participants ne sont pas très enthousiastes de passer par les ingando. Les ingando sont perçus au début, comme un service militaire qui impose une forte discipline aux jeunes adolescents, finalistes du secondaire. Ils l'ont entendu de leurs ainés, ou ils l'ont entendu de ceux qui combattent la pratique des ingando ou tout simplement ils ont perdu tout intérêt pour les ingando à cause de la nature et les conditions dans lesquelles les ingando se passent.

\section{- Les ingando sont un cadre d'éducation civique et d'enseignement d'une prétendue « vraie histoire».}

Bien qu'ils redoutent les ingando avant et au début des ingando, les participants en sortent satisfaits, du moins à les entendre directement à la fin de la formation. Dans ce sens les ingando sont perçus comme un lieu de transformation des représentations sociales. Ils affirment que les ingando leur ont apporté beaucoup : Premièrement, c'est « la vraie histoire » à laquelle ils opposent la «fausse et la meurtrière » d'avant génocide qui prend racine dans la période coloniale et s'est cristallisée pendant les deux premières républiques.

Les participants aux ingando sont généralement impressionnés par ce dispositif qui parvient à réunir Hutu, Tutsi et Twa, des élèves du nord et ceux du sud, de l'est et de l'ouest, les enfants des riches et ceux des pauvres, des rescapés et des descendants des bourreaux. Dans ce cas les ingando sont des cadres favorables pour le rapprochement entre les communautés au lendemain d'un conflit. A la fin des ingando, les participants ne sont pas forcément unis et réconciliés entre eux, mais ils en sont à une étape importante de la cohabitation pacifique.

Les ingando sont également perçus par la majorité de nos enquêtés comme un lieu privilégié d'éducation à l'unité et à la réconciliation nationale et un cadre d'enseignement de "la vraie histoire ». Par rapport à ces deux éléments sur lesquels les enquêtés ont exprimé leur satisfaction, nous pouvons nous interroger sur le fondement de leurs perceptions. Il est vraie que le rapprochement entre Hutu et Tutsi serait impératif, et qu'une identité rwandaise serait porteuse des chances de faire émerger l'unité nationale en déconstruisant les identités ethniques qui se sont révélées mortifères. Cependant, il ne faudrait pas perdre de vue que la diversité n'est pas forcément incompatible avec l'unité et la réconciliation ne se confond pas non plus avec un discours unitariste, elle procède autrement et requiert d'autres mécanismes spécifiques. La réconciliation procède autrement. ${ }^{51}$

Par rapport à l'enseignement de l'histoire, la recherche a montré que les participants aux ingando semblent avoir découvert la «vérité historique » longtemps dissimilée ou falsifiée. La véracité de cette version officielle ne tient pas seulement à sa conformité à la réalité, mais aussi par ce qu'elle met en avant l'unité entre les Rwandais, contrairement aux enseignements de l'histoire pendant la colonisation et les deux premières républiques.

\footnotetext{
${ }^{50}$ Nous reviendrons sur ces écarts dans les lignes qui suivent.

${ }^{51}$ Lire la thèse de SHYKA MUGABE A., Op.Cit, 2009, pp.76-106.
} 
Plus tard, le doute s'installe. Après les ingando, les esprits ne sont pas forcément lavés des anciennes vérités partielles et des idéologies négatives acquises dans les différents milieux, dont la famille, l'école, les livres, les médias et l'internet. Si le «lavage des cerveaux » n'a pas été exclu des intentions des orateurs ou de l'homme politique, en tout cas les résultats montrent bien que les esprits sont loin de répéter naïvement tous les enseignements reçus. Ils s'en accommodent parfois et à bien d'égards, mais, ils s'en écartent aussi ou contredisent carrément la version officielle.

\section{- Les ingando, une approche «top-down »}

Depuis la conception de ce programme, les ingando sont conçus d'en haut bien que le débat autour de la conduite de ces activités semble faire l'unanimité des participants aux dialogues nationaux. Ceux-ci sont organisés au niveau du sommet de l'Etat.

En interrogeant le processus de décision dans la mise en œuvre de la politique d'unité et de réconciliation en général et des ingando en particulier, en interrogeant les participants sur les méthodes d'enseignement dans les ingando, nous avons constaté que le processus de prise de décision va généralement du haut en bas, (top-down process). Il est vrai que plusieurs sommets et dialogues nationaux ont été organisés au siège de la présidence et au parlement, mais le cadre reste politiquement fort encadré, au point de lui enlever le caractère strictement démocratique. Le rapport d'évaluation des activités des ingando en 2007 soulignait l'importance des mécanismes locaux de mobilisation des communautés à la participation aux ingando, d'une part et impliquer les leaders locaux dans l'organisation des activités d'ingando. ${ }^{52}$ L'implication ne se situerait pas seulement dans l'exécution du programme, au contraire, les communautés locales devraient participer à la conception des programmes qui tiennent en compte les particularités de chaque région. C'est à cette condition que des stratégies et des actions concrètes devront répondre aux besoins en éducation civique au niveau des populations.

Dans la transmission des leçons enfin l'approche effectivement utilisée dans les ingando démontre d'une participation marginale des participants aux séances de formation. La leçon se transforme presque en une séance d'information. Les thèses officielles sont constamment réitérées et les participants posent des questions auxquelles ils reçoivent des réponses rarement ou jamais remises en question.

\section{- Ingando et les interférences identitaires et les intérêts personnels des orateurs}

Les résultats de nos recherches montrent que la manière dont les enseignements sont dispensés dans les ingando est fortement influencée par les interférences identitaires et les intérêts personnels des orateurs. Même si le contenu peut être le même, la manière d'enseigner peut varier selon que l'on est Hutu ou Tutsi ou perçu en tant que tel, ou selon que l'on est officier militaire ou civil et la manière peut varier également selon que l'on est membre du FPR ou d'un autre parti. Ces variations sont très difficilement perceptibles, mais significatives.

Premièrement, tous ces orateurs ont différentes expériences et ils ont leur manière personnelle d'interpréter le passé et le génocide, mais leur rôle de politicien ne leur permet pas forcément de se distancier de la version officielle pour faire face aux flux des questions qui paraissent

\footnotetext{
${ }^{52} \ll$-To determine the role of the implementing department in organizing and motivating the Ingando beneficiaries; -To mobilize and support local political and opinion leaders in promotion of Ingando activities at the grass-root and community level" Cfr CNUR, Impact of the solidarity camps/INGANDOs on Unity and Reconciliation of Rwandans", 1996 - 2006, Kigali 2007.
} 
controversées. Les uns se sentent plus libres de fournir des exemples puisés dans leur propre passé personnel, d'autres s'efforcent d'être convaincants sans y parvenir, d'autres encore sont convaincants, seulement par leur rang d'officier ou par leurs titres académiques. Mais, tous se rejoignent dans la seule manière d'interpréter l'histoire et l'idéal d'unité et de réconciliation.

\section{- Les ingando transmettent une seule « vérité unique et absolue ».}

Que vérité officielle transmise soit fondée ou pas, cette version unique, la « vraie » et la plus réconciliatrice, peut ne pas forcément correspondre à la réalité des faits historiques, mais elle est celle véhiculée par l'Etat, il faut à tout prix s'en convaincre. Cette vérité, une, unique et vraie est dotée d'une vision d'unité et de réconciliation. C'est ce qui fait la différence de fond par rapport à l'ancienne, fausse et meurtrière. C'est pourquoi, elle doit prendre place dans les esprits et transformer les comportements. Cette mémoire est, dès lors, sélective et mise au service d'un idéal commun.

\section{- Absence ou insuffisance de mécanisme d'accompagnement}

A part les clubs d'unité et de réconciliation qui réunissent les anciens participants aux ingando, aucun autre mécanisme d'accompagnement n'a été prévu jusqu'à ce jour ${ }^{53}$. A la fin des ingando, les participants se regroupent volontairement dans des clubs d'unité et de réconciliation, dans les universités et les écoles pour tenter de pérenniser l'idéal d'unité et de réconciliation et surtout pour disséminer les valeurs y relatives autour d'eux. Or, les participants aux ingando redoutent la résurgence des idéologies dans leurs esprits. C'est pourquoi à la fin de chaque édition des ingando, les participants ne ratent pas l'occasion de demander aux autorités d'étendre les ingando à toutes les couches sociales. La plupart du temps, les participants demandent que la formation soit continue sous d'autres formes. Cette crainte de voir ressurgir en eux l'idéologie négative présage la fragilité des connaissances acquises.

\section{- Continuité et discontinuité entre l'unité nationale, l'identité nationale et la mémoire collective}

L'analyse de l'action de l'Etat dans la gestion du passé a montré qu'il existe une discontinuité importante dans le processus de mise en œuvre des politiques publiques en rapport avec la gestion du passé au Rwanda. De la conception du dispositif ingando à la mise en place de la CNUR, du contenu du programme et de la politique qui le régit : des écarts très significatifs sont visibles entre les objectifs écrits dans la Constitution et dans la politique de la CNUR et les objectifs effectivement réalisés lors des ingando. Les modalités de mise en œuvre de cette politique des ingando ne suivent aucun plan préétabli. Cela explique, en partie,

\footnotetext{
53 A part ces clubs d'unités et de réconciliation, il existe deux autres mécanismes qui ne s'adressent pas expressément aux jeunes étudiants qui ont fait ingando, à savoir le système d'alerte et de prévention et les forums des enseignants. Ces deux mécanismes servent d'outils d'évaluation et de suivi du processus d'unité et de réconciliation au niveau de la base. Avec le système d'alerte et de prévention précoce plus de 416 rapports issus des districts et des provinces sont centralisés au niveau de la CNUR chaque mois. Ils ont pour objectif de relever et de prévenir les obstacles au processus d'unité et de réconciliation. Ces mécanismes permettent de relever les particularités des transformations des conflits dans chaque région. Tandis que les forums des enseignants font rapport aux agents de la CNUR au niveau des districts sur les obstacles à l'unité et la réconciliation. Dix ans après la mise en place d'ingando, en 2007, un rapport d'évaluation des ingando avait insisté sur l'absence d'un système d'évaluation en ces termes." - To initiate a strategic insight on the need assessment for Ingando longterm perspective - To exchange on the possibility to design "NATIONAL STANDARDS FOR INGANDO FOCUSED CIVIC EDUCATION AND SOCIAL DIALIGUE”, in general and in direction of the schools and local communities, in particular.- To provide a "National Performance Review Mechanism (NPRM) focusing on the Evaluation \& Monitoring and Reward system; - To evaluate Ingando sustainability and new development in perspective of a global Ingando Reform". A lire dans, CNUR, Impact of the solidarity camps/INGANDOs on Unity and Reconciliation of Rwandans". 1996-2006, Kigali, 2007, pp. 89-92.
} 
l'absence de mécanismes concrets, réguliers, homogènes et rigoureux pour évaluer les ingando au niveau de leur impact sur les représentations sociales

Du point de vue de la cohérence de fond, il est sans doute vrai qu'une nation requiert l'unité nationale à travers une identité nationale et une communauté des mémoires. Cependant entre les trois éléments du processus de «nation building » il y a des écarts de sens, bien que les trois approches se complètent et se rejoignent dans une même stratégie globale de reconstruction de la société post conflit.

L'unité nationale ne suppose pas forcément une fusion identitaire. Elle peut procéder par une intégration des différentes identités autour d'un objectif commun et autour d'une vision commune du passé conflictuel qui prend en compte les divergences. Que ces identités soient objectivement différentes ou subjectivement construites, elles n'influencent pas moins le comportement des individus. On peut choisir de les intégrer comme des réalités historiques à prendre en compte dans le processus de reconstruction, ou du moins les déconstruire en encourageant les catégories sociales à donner la chance à une identité nationale qui est plus porteuse des germes d'unité nationale. La mémoire collective, quant à elle, ne s'imposera pas d'en haut, il faudrait à tout prix prendre en compte les divergences inhérentes à toute société post-conflits. Elle ne se réduit pas à une simple addition des différentes interprétations du passé, ni à une mémoire officielle imposée d'en haut, elle résulte au contraire d'un travail de mémoire.

Tous ces thèmes nécessitent une articulation rigoureuse. Cette approche de nation building est sans doute la mieux indiquée pour la reconstruction sociale, il faut en assumer les exigences et les responsabilités.

- Il y a des écarts significatifs entre mémoire officielle et représentations sociales,

Les représentations sociales du passé et du génocide chez nos enquêtés ne rejoignent pas forcément la mémoire officielle transmise dans les ingando. Nos conclusions rejoignent celles de l'IRDP qui avait conclu que les écarts se situaient moins au niveau des faits mêmes qu'à leur sens. Le rapport de recherche sur la cohésion sociale et la question ethnique affirme ce qui suit: "l'écriture de l'histoire exige que l'on se préoccupe d'établir suffisamment les faits historiques avant de les interpréter. En ce qui concerne le Rwanda, il importe de reconnaître qu'en plus des questions historiques controversées relatives à plusieurs domaines et qui constituent une partie infime de la réalité historique, il existe des faits dont l'existence et l'interprétation sont communément admises ${ }^{54}$.

En effet, l'écart entre la mémoire officielle et les représentations sociales du passé et du génocide est perceptible à travers les points de divergence, non pas sur la réalité des faits mêmes, mais sur le sens que l'on donne à ces derniers. D'où, la nécessité de repenser un travail de mémoire en commençant par rechercher les points de divergence et de convergence d'interprétation de l'histoire nationale.

- La période coloniale est interprétée comme un temps glorieux, une nation perdue dans laquelle Hutu et Tutsi vivaient en parfaite cohésion. Les conflits de nature sociale ne pouvaient pas manquer, mais ni l'un ni l'autre n'avait jamais eu l'intention d'exterminer l'autre. Cette vision fait l'unanimité de la plupart de nos enquêtés, mais qui ne manquent pas d'interroger en profondeur l'origine de ces peuples et la nature

\footnotetext{
${ }^{54}$ IRDP, Op.Cit, 2009.
} 
des relations sociales. Certaines catégories sociales dénoncent l'insuffisance du mythe de Gihanga pour expliquer les origines d'un peuple. Ils veulent comprendre la nature des relations entre les catégories Hutu et Tutsi avant la colonisation.

A ce propos, la recherche a montré que l'ubuhake reste un point d'achoppement qui reste en veille dans les esprits de certains Rwandais aux idéologies «pro-hutu ». L'ubuhake est révélé comme le plus grand silence assourdissant entretenu dans la mémoire officielle transmise dans les ingando. Quand on en parle, c'est pour principalement en démontrer le bon côté, en reconnaissant très peu ses méfaits sur les relations sociales au Rwanda.

Par rapport à l'argument de pouvoir, cette période est encore perçue comme ayant favorisé l'élite Tutsi au pouvoir, contre « une majorité Hutu asservie sous la monarchie »

Il faut dire qu'il y a de nombreux points de rencontre entre les différentes formes d'interprétation, il suffit de faire ressortir ces différentes interprétations pour y trouver un sens commun qui permet de guérir certaines de leurs blessures. Que celles-ci soient réelles ou subjectives, elles n'influencent pas moins leur mémoire collective. En montrant avec preuve à l'appui, que des Tutsi de la basse classe pouvaient subir les dérives de l'ubuhake, on éviterait la dichotomisation identitaire par rapport à la pratique de l'ubuhake au Rwanda.

Les divergences très peu significatives apparaissent encore au niveau de la responsabilité coloniale dans le développement des conflits rwandais. Dans la mémoire officielle la colonisation est l'argument massue pour expliquer les origines des conflits rwandais. L'arrivée des colons est un moment clé de rupture dans les relations sociales au Rwanda. Cependant quelques voix s'élèvent timidement en dénonçant les conflits inhérents aux sociétés traditionnelles et la responsabilité des Rwandais dans leurs propres conflits. La colonisation, oui, mais pas seul responsable de nos malheurs : Quelle est la part des Rwandais dans leur propre malheur avant les deux premières républiques? Cet argument veut examiner les responsabilités sociales et politiques sous la monarchie au Rwanda. Le manuel officiel présente unilatéralement la responsabilité coloniale pendant cette période qui par ailleurs a précédé les deux premières républiques établies sur fond d'une « révolution » dite sociale

\section{- «En 1959, il n’y a pas eu révolution, il y’a eu, certes, un changement social meurtrier $»$,}

Telle est la conclusion principale de la leçon en rapport avec les relations sociales, dispensée dans les ingando. Pas de révolution, plutôt changement social qui n'a profité qu'aux colonisateurs et aux élites Hutu au pouvoir. Elle n'a pas concerné tous les Rwandais, au contraire, elle a porté les Hutu extrémistes à tuer les Tutsi et à en chasser d'autres à l'extérieur du Rwanda. Dans la mémoire officielle, 1959 symbolise même le début du génocide poursuivi jusqu'en 1994. Dans la mémoire collective des Hutu, la révolution a bien eu lieu, «elle affranchit les Hutu de la servitude Tutsi ». Cet argument fut utilisé en 1994 pendant le génocide pour mobiliser les masses Hutu à se lever contre les Tutsi qui risqueraient de les ramener sous le joug de l'ubuhake. Dans ce sens, la crainte de voir ressurgir l'ubuhake avec le retour des Tutsi au pouvoir constitue une justification du génocide. Les arguments affluent dans ce sens pour affirmer que la révolution a eu lieu et sa raison d'être par ce qu'elle a renversé la monarchie Tutsi, elle a chassé les colonisateurs et a installé une république sur base de la majorité Hutu. Les mêmes arguments sont mobilisés quand les enquêtés s'expriment sur le bilan de l'indépendance de 1962. Ces représentations sociales sont d'actualité et ne disparaissent pas encore des esprits de nos enquêtés malgré leur participation aux ingando. 
- Les deux premières républiques constituent indistinctement une période noire de l'histoire nationale. Elles portent la responsabilité d'avoir planifié systématiquement un génocide contre les Tutsi. Plusieurs preuves sont irréfutables, la première et la deuxième ont installé la division, l'ethnocratie basée sur la divisions, la discrimination régionale, les quotas ethniques dans les écoles et la fonction publique, ...

Ce point de vue convainc bien évidement la majorité des Rwandais et les faits parlent d'euxmêmes. Cependant la recherche a montré que le bilan des deux premières républiques n'était pas que négatif dans les représentations sociales de nos enquêtés. Ils distinguent les réalisations économiques des réalisations politiques et sociales. Certains expriment la nostalgie d'un paradis perdu dans lequel le Hutu était au pouvoir. Ils en gardent la bonne mémoire au point de lui attribuer l'essor économique que le FPR au pouvoir n'a fait que poursuivre : Les deux premières républiques ont développé les régions du nord et du sud, elles ont construit des routes, des écoles, des bâtiments qui abritent les différents ministères, elles ont développé la culture du café et du thé, ont construit l'aéroport international de Kigali,...Au final, ils reconnaissent tout de même que ce bilan économique était malheureusement accompagné d'une politique de discrimination contre les Tutsi et contre les ressortissants des autres régions que le sud et le nord d'où venaient les élites au pouvoir. Les inégalités sociales se sont renforcées au fil des années et le génocide a été progressivement et systématiquement mis en place jusqu'à son apogée en 1994. Les relations sociales sont donc le fondement principal du bilan négatif des deux premières républiques. La reconnaissance d'un bilan, quelque peu positif, des réalisations économiques passées modifierait les représentations sociales du passé ? Cette reconnaissance pourrait-elle minimiser le génocide et sa préparation systématique. ? Reconnaitre le bilan économique et en établir les preuves apaiseraient probablement la culpabilité de certains Hutu qui pensent n'avoir été bons à rien

- Le génocide est une réalité aujourd'hui difficilement réfutable, mais il reste des points de divergences dans les différentes représentations sociales. D'aucuns nient la réalité de ce fait, cependant, les divergences apparaissent quand il s'agit d'expliquer ses causes, de tenter de le qualifier, de distinguer les bourreaux des victimes et de la nature de la mémoire que l'on en garde. Cette difficulté tient au contexte et à la nature même de ce génocide.

Par rapport à la qualification du génocide, la mémoire officielle a pris du temps pour se stabiliser. Les événements d'Avril à juillet 1994 ont été d'abord été qualifiés de "génocide ethnique et massacres » traduit du Kinyarwanda, "Itsembabwoko Itsembatsemba ». Cette qualification a été opérationnelle dans les discours et dans les documents depuis 1995 jusqu'à la veille du dixième anniversaire en 2004. Depuis 2003, la nouvelle constitution du Rwanda a consacré le mot "jenoside» pour dissiper la confusion entre massacre et génocide. Depuis 2004, la qualification était réduite à "génocide des Tutsi », comme on dirait «génocide des juifs ». Quatre ans plus tard, la qualification semblait encore confuse. En disant " génocide des Tutsi », la distinction entre celui qui l'a commis et celui qui l'a subi semblait confuse. Depuis 2008, une commission ad hoc, sous l'égide d la CNLG, a tranché en donnant à ces événements la qualification encore en vigueur: «génocide commis contre les Tutsi ».

Ces changements majeur et porteur de sens n'ont pas épargné les esprits de nos enquêtés malgré que notre enquête s'est passée principalement au lendemain de l'adoption de la nouvelle qualification. Au cours de cette recherche, il a été évident que tous les Rwandais ne rejoignent pas encore cette manière de qualifier le génocide. Certains dénoncent le silence autour des victimes Hutu sans méconnaître, ni nier le caractère exclusif de la victime Tutsi 
comme principale cible du génocide. D'autres vont plus loin en revendiquant la reconnaissance officielle des Hutus morts avant, pendant et après le génocide. D’autres, enfin, préviennent les décideurs politiques en disant que cette qualification actuelle risque, à la longue, de consolider un repli identitaire des Hutu contre les Tutsi, ce qui serait contre la ligne actuelle d'unité et de réconciliation nationale.

Pourquoi évoquer à tout prix la victime Tutsi dans le processus global de reconstruction nationale qui ne reconnait pas officiellement le fondement objectif des identités ethniques sur base desquelles le génocide a été commis ? La mémoire devra-t-elle suivre une autre logique qui ne soit pas celle de la reconstruction sur base de l'identité nationale ? Y'a-t-il contradiction entre la mémoire du génocide qui doit faire explicitement référence aux identités Hutu, Tutsi et Twa et le modèle de reconstruction qui fait fusion des identités dans une seule, c'est-à-dire, la «rwandité » ou « ubunyarwada »?

Nous sommes bien conscients de la délicatesse de ce débat, mais c'est cela même le travail de mémoire qu'il faudrait courageusement aborder. Ce débat soulève deux grandes questions qui referont toujours surface dans toute tentative de travail de mémoire. L'une remet en question le modèle de reconstruction nationale qui se donne l'ambition de déconstruire les identités ethniques dans le contexte du Rwanda. L'autre se rapporte à la question du statut du Hutu « juste ${ }^{55}$ pendant le génocide.

D'une part, le rescapé revendique son identité de «Tutsi » sur base de laquelle il a perdu les siens et le Tutsi, absent pendant le génocide fait valoir la fierté d'être considéré comme tel. Comment parler de la mémoire sans parler des «Tutsi », principales cibles et victimes du génocide?

D'autre part les Hutu qui ont la honte et la culpabilité d'être perçus comme tels ou d'autres Hutu qui restent fiers de leur identité. Comment parler du génocide sans évoquer l'identité au nom de laquelle ce génocide a été commis. Pourtant, la question de la victime hutu resurgit sans cesse dans les représentations sociales. Comment envisager la justice, la vérité sur le passé et la réconciliation comme des préalables d'une mémoire véritablement nationale ?

Toutes ces questions affectent inévitablement la politique de la mémoire du génocide au Rwanda. Elles sont encore loin de faire l'unanimité de tous les Rwandais.

Il est donc plus qu'évident que la mémoire collective sera fonction du temps au lieu de prétendre avoir transformé les représentions sociales dans une formation d'ingando qui n'a duré que trois semaines à peine.

La reconstruction de la société rwandaise après le génocide est un impératif, on le constate à travers les différents points de vue qui s'expriment sur les modèles de reconstruction de la société. La mémoire est un impératif, un devoir, un besoin et un chantier qu'il faut à tout prix aménager. Mais les divergences apparaissent dans la manière d'évoquer le passé et surtout dans la manière de réinterpréter le passé et la gestion de la question identitaire. La reconstruction de la société sur base d'une identité nationale ne permet pas pour certains d'évoquer sans tomber dans l'ambiguïté le passé marqué par les ethnies. Pourtant, certains ne

\footnotetext{
${ }^{55}$ ROSOUX V., «La figure du Juste au Rwanda : héros, traître ou inconnu ? », Revue internationale des sciences sociales, 2006/3 $\mathrm{n}^{\circ} 189$, p. 530, consulté en ligne sur, http://www.cairn.info/revue-internationale-des-sciences-sociales-2006-3-page-525.htm, le 20 juin 2012.
} 
doutent pas de la nécessité de mobiliser des mécanismes plus inclusifs, autres que ceux qui ont conduit au génocide, c'est-à-dire une identité nationale. La thèse démontre à la suite d'autres travaux réalisés par le sénat de la république, l'IRDP de la CNLG et de la CNUR qu'un travail plus approfondi sur ces questions reste souhaitable.

\section{Perspectives d'un travail de mémoire : quelques suggestions.}

L'étude de cas sur les ingando en tant que dispositif officiel à travers lequel le Rwanda transmet une certaine mémoire officielle confère inévitablement à cette réflexion une dimension prescriptive.

A cet égard, nous suggérons que ce programme soit étendu à toutes les couches sociales et surtout qu'il se formalise à travers l'enseignement de l'histoire au niveau du primaire, secondaire et universitaire. Dans ce cas, les ingando seraient limités dans le temps et devraient à un certain moment céder la place à un programme plus structurée et renouvelée selon les impératifs présents et en conformité avec la politique de la mémoire au Rwanda.

- En attendant la formulation d'un programme formel d'enseignement de l'histoire qui se fait trop attendre, le manuel actuel de formation devrait évoluer et être adapté aux impératifs du moment.

- L'enseignement de l'histoire requiert non seulement des méthodes rigoureuses de critique historique, mais aussi du personnel compétent sélectionné sur base de critères objectifs.

- Le passage des ingando à l'itorero ${ }^{56}$, deux structures différentes par leur philosophie et leur objectif, a été tellement brusque que la confusion entre les deux règne dans les représentations sociales. Pour les uns les deux sont utilisés l'un à la place de l'autre et pour d'autres, les ingando n'existent plus, il n'existe que itorero. Les deux structures ne s'équivalent pas et ne sont pas, non plus, incompatibles. Par contre il faudrait une forte campagne de sensibilisation sur les deux mécanismes d'éducation civique et définir clairement les limités entre les deux mécanismes de façon qu'ils soient complémentaires.

- Le risque d'itorero est de diluer la profondeur des enseignements dispensés dans les les ingando depuis plus 18 ans dans des slogans et des discours moralisateurs sans pouvoir s'assurer de leur consistance et leur durabilité.

- Il est recommandable qu'un travail de grande envergure soit réalisé et que des débats plus ouverts et cadrés soient organisés pour amener les catégories sociales rwandaises à participer à cet édifice d'une mémoire collective nationale.

En conclusion, le dispositif a servi certes à la stabilisation de la société et au rapprochement entre les catégories sociales dont les lignes de séparations étaient idéologiquement très significatives au lendemain du génocide. Le dispositif lui-même tient son originalité, d'abord, au fait qu'il a puisé dans la tradition rwandaise et s'est adapté au contexte rwandais ${ }^{57}$, ensuite

\footnotetext{
${ }^{56}$ En effet, depuis 2007, les deux mécanismes d'ingando et d'itorero cohabitent et sont organisés par la CNUR Dans l'itorero, on enseigne également l'histoire du Rwanda, mais dans le but d'y retrouver les valeurs partagées par tous les Rwandais. Ils s'adressent à toutes les catégories sociales rwandaises. Les premiers, organisés en mai 2008, ont été consacré aux enseignants de l'école primaire, aux infirmiers, aux motards, aux secrétaires exécutifs des secteurs, aux élèves en fin du secondaire,...

Par ailleurs, plusieurs éléments de forme et de fond montrent qu'il y a rupture entre les deux mécanismes. Même si les deux sont des dispositifs de rééducation, l'itorero vise un objectif plus global, car il vise à mobiliser les valeurs et les interdits partagés par tous les Rwandais pour un développement et le bien être intégral de l'homme Rwandais

57 «Home-grown solution»
} 
au fait qu'il a rassemblé des catégories sociales aux multiples blessures au lendemain du génocide.

Ce dispositif a le mérite d'avoir initié des cadres de discussions sur de grandes questions nationales, d'avoir amené les anciens ennemis autour d'une vision commune dispensée par les autorités officielles. Ils ont grandement contribué au rapprochement entre les groupes, mais le travail de mémoire reste à faire. Ce travail qui déconstruit les représentations sociales négatives ne peut que s'inscrire dans le temps. A part la variable temps, cette réflexion a montré la nécessité d'une révision des contenus, des méthodes et la prise en compte des interférences identitaires de ceux qui sont en charge de transmettre cette mémoire officielle. En attendant, il est important de ne pas prétendre naïvement que la mémoire collective résulterait d'une seule interprétation du passé, au contraire, la mémoire officielle devrait s'assurer d'une cohérence interne, des compétences requises et d'un contenu incluant le débat sur les différentes interprétations du passé et de la mémoire du génocide en vue d'en arriver à une trame commune plus ou moins acceptable par tous. Une mémoire collective nationale ne sera pas le simple produit des représentations officielles du passé national. Valérie Rosoux avait montré la nécessité d'une prise en compte des différentes interprétations du passé ${ }^{58}$ et proposait également d'inscrire l'action dans le temps. Partant de toutes ces perspectives, nous proposons à notre tour une perspective d'émergence d'une mémoire collective nationale véritablement inclusive qui requiert la mise en relation trois principaux éléments et trois acteurs à savoir la mémoire officielle ou l'Etat, les représentations sociales (la population), la recherche scientifique (chercheurs) et le soutien de la communauté internationale dont la Belgique, la France, les Etats-Unis et la Grande Bretagne) ${ }^{59}$.

\footnotetext{
${ }^{58}$ ROSOUX V., "La gestion du passé au Rwanda : ambivalence et poids du silence ", Genèses, 2005/4 n 61, p.40, (p. 28-46).

${ }^{59}$ Cette perspective de travail de mémoire a été largement développée dans notre thèse de doctorat portant sur cette même thématique de politique de mémoire (à paraitre). Thèse disponible dans les bibliothèques de l'académie Louvain et en ligne sur :
} 


\section{Références bibliographiques}

1. ABRIC J-C., «Les représentations sociales : aspects théoriques », in ABRIC J-C. s/dir.), Pratiques sociales et représentations, PUF Paris, 1994

2. ADEKUNLE J., Culture and Customs of Rwanda, Greenwoods Press, Westport, 2007

3. ALCAUD D., "Sociologie des arts et de la culture et politique culturelle : l'évolution des répertoires d'action en Italie », dans GIREL S., (s/dir..), Sociologie des arts et de la culture. Un état de la recherche, L'Harmattan, Paris, 2006

4. AMSELLE et M'BOKOLO, (s/dir.), Au cour de l'ethnie. Ethnies, tribalisme et Etat en Afrique, La Découverte, Paris, 1985.

5. AMSELLE J-L. et M'BOKOLO E., «Au cœur de l'ethnie. Ethnies, tribalisme et Etat en Afrique, La découverte, Paris, 1985.

6. ANSART A., «Mémoire collective», dans Encyclopaedia Universalis, http://www.universalis.fr/encyclopedie/maurice-halbwachs/, le 2 Juillet 2012.

7. ARBORIO A.M., FOURNIER P., L'Enquête et ses méthodes. L'Observation directe, ( $2^{\mathrm{ème}}$ édition), Arman Colin, Paris, 2008.

8. LASCOUMES, P., et LE GALÉS, P., Gouverner par des instruments, Presses de Sciences po, Paris, 2004

9. LATOUR B., Petites leçons de sociologie des sciences, Seuil, Paris, 1996.

10. MAALOUF A., Les identités meurtrières, Grasset, Paris, 1998

11. LAVABRE M-C., Le fil rouge Sociologie de la mémoire communiste, Presses de la Fondation nationale des sciences politiques, 1994.

12. ROSOUX V. Les usages de la mémoire dans les relations internationales. Le recours au passé dans la politique étrangère de la France à l'égard de l'Allemagne et de l'Algérie. De 1962 à nos jours, Bruylant, Bruxelles, 2001

13. RUTEMBESA F., SEMUJANGA J., SHYAKA A., (s/dir), Rwanda. Identité et citoyenneté, Editions de l'Université Nationale du Rwanda, Butare 2003

14. SEBASONI S., Les origines du Rwanda, l'Harmattan, Paris, 2000

15. SEBASONI S., Reconstruire une nation, Ed. Rwandaise, Kigali, 2007

16. SEMUJANGA J., Récits fondateurs du drame rwandais. Discours social, idéologies et stéréotypes, Harmattan, Paris, 1998

17. ROSOUX V., «La figure du Juste au Rwanda : héros, traître ou inconnu ? », Revue internationale des sciences sociales, 2006/3 $\mathrm{n}^{\circ} 189$, p. 530, consulté en ligne sur, http://www.cairn.info/revue-internationale-des-sciences-sociales-2006-3-page$\underline{525 . h t m}$, le 20 juin 2012.

18. ROSOUX V., «La gestion du passé au Rwanda : ambivalence et poids du silence », Genèses, Sciences Sociales, ${ }^{\circ}$ 61, 2005/4

19. ROSOUX V., «La réconciliation franco-allemand: crédibilité et exemplarité d'un «couple à toute épreuve »? », dans Cahiers d'histoire. Revue d'histoire critique,(en ligne), 100, 2007, mis en ligne le 01 janvier 2010, consulté le11 mars 2011, p.2.

20. ROSOUX V., «Le Rôle de la mémoire en politique étrangère. Essai de théorisation », dans, TANGY. DE WILDE et L. SPETSCHINSKY L., Les relations entre l'UE et la Fédération de Russie, UCL, 2000. En ligne sur http://www.uclouvain.be/cps/ucl/doc/euro/documents/Rosoux__ue_russie.pdf ， 12 Décembre 2012

21. ROSOUX V., «Rwanda, l'impossible «mémoire nationale », dans BAUSSANT T M. et PETER J.P., « Mémoires plurielles, mémoires en conflit », Ethnologie française, 2007/3 Vol. 37, pp.409-415.

22. ROSOUX, V., «Les usages du passé dans la politique étrangère de la France », dans ANDRIEU C., LAVABRE M-C., TARTAKOWSKY D., Politiques du Passé, 
Usages politiques du passé dans la France contemporaine, Publication de l'Université de Province, 2006, p.177.

23. LAVABRE, M-C, «Du poids et du choix du passé. Lecture du syndrome de Vicy », dans PESCHANSKI D., POLLAK M., ROUSSO H., (s/dir), Histoire politique et sciences sociales, Edi complexes, Paris, 1991

24. LAURE de VULPIAN, Rwanda. Un génocide oublié ? Un procès pour mémoire, Ed. Complexe, Bruxelles, 2004

25. LAVABRE M-C., Le fil rouge Sociologie de la mémoire communiste, Presses de la Fondation nationale des sciences politiques, 1994.

26. LAVABRE, M-C, «Du poids et du choix du passé. Lecture du syndrome de Vicy », dans PESCHANSKI D., POLLAK M., ROUSSO H., (s/dir), Histoire politique et sciences sociales, Edi complexes, Paris, 1991

27. NSANZUBUHORO N. E., «Figure et identité de la victime à travers l'Association des Etudiants et Elèves Rescapés du Génocide, (AERG), dans «Génocide: Les figures de la victime », paru dans la revue La pensée et les Hommes, n85, 2012

28. NSANZUBUHORO N.E. « La gestion politique de la mémoire du génocide au Rwanda : Mémoire officielle face aux représentations sociales». Dans Cahiers de Sciences politiques de l'ULg, Cahier $\mathrm{n}^{\circ} 21$ disponible en ligne sur http://popups.ulg.ac.be/csp/document.php?id=568, consulté le 2 Octobre 2012.

29. SEMUJANGA J., «Formes et usages des préjugés dans le discours social du Rwanda », in RUTEMBESA F., SEMUJANGA J., SHYAKA A., Rwanda. Identité et citoyenneté, Ed de l'UNR, Butare 2003

30. SENAT DE REPUBLIQUE DU RWANDA, (S/dir d'Anastase SHYAKA), Rwanda. Idéologie du génocide et stratégies de son éradication, Kigali, 2006

31. SHYAKA A, (commanditée par la CNUR), Origines, Développement et Stratégies de sortie Etude commandée par La Commission Nationale pour l'Unité et la Réconciliation, Kigali, 2006

32. SHYAKA A., «Refondation de la rwandité : stratégie de résolution des conflits, de démocratisation et de développement durable du Rwanda», dans RUTEMBESA F, et al ii, Identité et Citoyenneté, Butare, UNR, 2003

33. CNUR, Rapport Anuel des activités de la CNUR. Période allant de Mars 1999 à Juin 2000, (Version française), Kigali, 2000.

34. CNUR, Rapport des activités de la CNUR : Année 2001, Kugali, Mai 2002

35. CNUR, Rapport d'évaluation du processus d'unité et de réconciliation nationale : 23 novembre 2001, Kigali, Juin 2002.

36. CNUR, Impact of the solidarity camps/INGANDOs on Unity and Reconciliation of Rwandans" 1996 - 2006, Kigali, 2006.

37. CNUR, Social cohesion in Rwanda. An opinion survey. Results 2005-2007, Kigali 2007.

38. SHYAKA A. (consultant), The Rwandan Conflict: Origin, Development, Exit Strategies. A Study ordered by The National Unity and Reconciliation Commission, Kigali, 2006.

39. MUGANGA (col. D/Comdt-Rwanda Military Academy Nyakinama), Report of the second ingando for Faculty staff of the national university of Rwanda, (NUR),

40. Harvard Human Rights Journal, Ingando Solidarity Camps: Reconciliation and Political Indoctrination in Post-Genocide Rwanda, on http://www.law.harvard.edu/students/orgs/hrj/iss 18/mgbako.shtml\#fn1

41. CNUR, Rapport des consultations nationales: initiative d'unité et de réconciliation au Rwanda, réalisées entre Août 2009- et Octobre 2010, Kigali 2010. 
42. Constitution de la République du Rwanda adoptée lors du Référendum du 26 mai 2003 tel que confirmé par la Cour Suprême dans son Arrêt $n^{\circ} 772 / 14.06 / 2003 \mathrm{du}$ 02/06/2003.

43. Government of Rwanda, Report on the Reflection Meetings Held in the Office of the President of the Republic from May 1998 to March 1999, Kigali, 1999.

44. La loi organique $\mathrm{n}^{\circ} 16 / 2004$, (en son article 34), portant organisation, compétence et fonctionnement des juridictions gacaca.

45. Loi organique du 19 juin 2004 portant organisation, compétence et fonctionnement des Juridictions gacaca chargées des poursuites et du jugement des infractions constitutives du crime de génocide et d'autres crimes contre l'humanité, commis entre le $1^{\text {er }}$ octobre 1990 et le 31 décembre 1994.

46. MIJESPOC, Politique sectorielle de la mémoire du génocide commis contre les Tutsi, Kigali, 2007, politique adoptée par le conseil des ministres du 12 Mars 2008.

47. MIJESPOC, Politique sectorielle de la mémoire du génocide des Tutsi, Kigali, 2008. 